CTP/TAMU-49/93

CERN-TH.7088/93

\title{
New Black Hole, String and Membrane Solutions of the Four-Dimensional Heterotic String*
}

\author{
M. J. Duff ${ }^{1}$, Ramzi R. Khuri ${ }^{2 \dagger}$, Ruben Minasian ${ }^{1}$ and Joachim Rahmfeld ${ }^{1}$ \\ ${ }^{1}$ Center for Theoretical Physics \\ Texas A\&M University \\ College Station, TX 77843 \\ 2 Theory Division, CERN \\ CH-1211, Geneva 23, Switzerland
}

\begin{abstract}
We present solutions of the low-energy four-dimensional heterotic string corresponding to $p$-branes with $p=0,1,2$, which are characterized by a mass per unit $p$-volume, $\mathcal{M}_{p+1}$, and topological "magnetic" charge, $g_{p+1}$. In the extremal limit, $\sqrt{2} \kappa \mathcal{M}_{p+1}=g_{p+1}$, they reduce to the recently discovered non-singular supersymmetric monopole, string and domain wall solutions. A novel feature is that the solutions involve both the dilaton and the modulus fields. In particular, the effective scalar coupling to the Maxwell field, $e^{-\alpha \phi} F_{\mu \nu} F^{\mu \nu}$, gives rise to a new string black hole with $\alpha=\sqrt{3}$, in contrast to the pure dilaton black hole solution which has $\alpha=1$. This means that electric/magnetic duality in $D=4$ may be seen as a consequence of string/fivebrane duality in $D=10$.
\end{abstract}

CTP/TAMU-49/93

CERN-TH.7088/93

November 1993

\footnotetext{
*Work supported in part by NSF grant PHY-9106593.

$\dagger$ Supported by a World Laboratory Fellowship.
} 


\section{Introduction}

In recent work [1], supersymmetric soliton solutions of the four-dimensional heterotic string were presented, describing monopoles, strings and domain walls. These solutions admit the $D=10$ interpretation of a fivebrane wrapped around 5,4 or 3 of the 6 compactified dimensions and are arguably exact to all orders in $\alpha^{\prime}$. In this paper, we extend all three solutions to two-parameter solutions of the low-energy equations of the four-dimensional heterotic string, characterized by a mass per unit $p$-volume, $\mathcal{M}_{p+1}$, and topological "magnetic" charge, $g_{p+1}$, where $p=0,1$ or 2 . We show them to be special cases of the generic black $p$-branes discussed in [2]. The "neutral" (as opposed to the "gauge" or "symmetric") solitons discussed in [1] are recovered in the extremal limit, $\sqrt{2} \kappa \mathcal{M}_{p+1}=g_{p+1}$, and are non-singular in the sense that the curvature singularity disappears when expressed in terms of the dual $\sigma$-model metric [2,3]. The two-parameter solution extending the supersymmetric monopole corresponds to a magnetically charged black hole, while the solution extending the supersymmetric domain wall corresponds to a black membrane. By contrast, the two-parameter string solution does not possess a finite horizon and corresponds to a naked singularity.

All three solutions involve both the dilaton and the modulus fields, and are thus to be contrasted with pure dilaton solutions. In particular, the effective scalar coupling to the Maxwell field, $e^{-\alpha \phi} F_{\mu \nu} F^{\mu \nu}$, gives rise to a new string black hole with $\alpha=\sqrt{3}$, in contrast to the pure dilaton solution of the heterotic string which has $\alpha=1$ [4 13]. It thus resembles the black hole previously studied in the context of Kaluza-Klein theories [14 [19,4,11] which also has $\alpha=\sqrt{3}$, and which reduces to the Pollard-Gross-Perry-Sorkin [16 18] magnetic monopole in the extremal limit. In this connection, we recall the recent paper of Holzhey and Wilczek [20], according to which $\alpha>1$ black holes behave like elementary particles!

The fact that the heterotic string admits $\alpha=\sqrt{3}$ black holes also has implications for string/fivebrane duality [21 23]. We shall show that electric/magnetic duality in $D=4$ may be seen as a consequence of string/fivebrane duality in $D=10$.

\section{The Solutions}

We begin with the two-parameter black hole. Inspired by the wrapping of a fivebrane around five of the six compactified dimensions $\left(x_{5}, x_{6}, x_{7}, x_{8}, x_{9}\right)$, it was shown in [1] that 
the tree-level effective action for the $D=10$ heterotic string may be reduced to the following four-dimensional form

$$
S_{1}=\frac{1}{2 \kappa^{2}} \int d^{4} x \sqrt{-g} e^{-2 \Phi-\sigma_{1}}\left(R+4(\partial \Phi)^{2}+4 \partial \sigma_{1} \cdot \partial \Phi-\frac{1}{4} e^{2 \sigma_{1}} F_{\mu \nu} F^{\mu \nu}\right),
$$

where $\mu, \nu=0,1,2,3$. Here $g_{\mu \nu}$ is the string sigma-model metric and $\Phi$ is the dilaton. In the case of toroidal compactification, with $N=4$ supersymmetry in $D=4, \sigma_{1}$ is a modulus field, $g_{44}=e^{-2 \sigma_{1}}$, and $F_{\mu \nu}=H_{\mu \nu 4}$ where $H=d B$ and $B$ is the string antisymmetric tensor. However, actions of this type also appear in a large class of $N=1$ supergravity theories [24]. The solution is given by

$$
\begin{aligned}
e^{-2 \Phi} & =e^{2 \sigma_{1}}=\left(1-\frac{r_{-}}{r}\right), \\
d s^{2} & =-\left(1-\frac{r_{+}}{r}\right)\left(1-\frac{r_{-}}{r}\right)^{-1} d t^{2}+\left(1-\frac{r_{+}}{r}\right)^{-1} d r^{2}+r^{2}\left(1-\frac{r_{-}}{r}\right) d \Omega_{2}^{2}, \\
F_{\theta \varphi} & =\sqrt{r_{+} r_{-}} \sin \theta
\end{aligned}
$$

where here, and throughout this paper, we set the dilaton vev $\Phi_{0}$ equal to zero. This represents a magnetically charged black hole with event horizon at $r=r_{+}$and inner horizon at $r=r_{-}$. The magnetic charge and mass of the black hole are given by

$$
\begin{aligned}
g_{1} & =\frac{4 \pi}{\sqrt{2} \kappa}\left(r_{+} r_{-}\right)^{\frac{1}{2}}, \\
\mathcal{M}_{1} & =\frac{2 \pi}{\kappa^{2}}\left(2 r_{+}-r_{-}\right)
\end{aligned}
$$

Changing coordinates via $y=r-r_{-}$and taking the extremal limit $r_{+}=r_{-}$yields:

$$
\begin{aligned}
& e^{2 \Phi}=e^{-2 \sigma_{1}}=\left(1+\frac{r_{-}}{y}\right), \\
& d s^{2}=-d t^{2}+e^{2 \Phi}\left(d y^{2}+y^{2} d \Omega_{2}^{2}\right), \\
& F_{\theta \varphi}=r_{-} \sin \theta .
\end{aligned}
$$

which is just the neutral (i.e. no Yang-Mills) version of the supersymmetric monopole solution [25 28,1] which saturates the Bogomol'nyi bound $\sqrt{2} \kappa \mathcal{M}_{1} \geq g_{1}$.

Next we derive a two-parameter string solution which, however, does not possess a finite event horizon and consequently cannot be interpreted as a black string. This is inspired by the wrapping of the fivebrane around four of the compactified dimensions $\left(x_{6}, x_{7}, x_{8}, x_{9}\right)$. The action is given by

$$
S_{2}=\frac{1}{2 \kappa^{2}} \int d^{4} x \sqrt{-g} e^{-2 \Phi-2 \sigma_{2}}\left(R+4(\partial \Phi)^{2}+8 \partial \sigma_{2} \cdot \partial \Phi+2\left(\partial \sigma_{2}\right)^{2}-\frac{1}{2} e^{4 \sigma_{2}} F_{\mu} F^{\mu}\right),
$$


In the case of the torus, $\sigma_{2}$ is the modulus field $g_{44}=g_{55}=e^{-2 \sigma_{2}}$ and $F_{\mu}=H_{\mu 45}$. A two-parameter family of solutions is now given by

$$
\begin{aligned}
e^{2 \Phi} & =e^{-2 \sigma_{2}}=(1+k / 2-\lambda \ln y), \\
d s^{2} & =-(1+k) d t^{2}+(1+k)^{-1}(1+k / 2-\lambda \ln y) d y^{2}+y^{2}(1+k / 2-\lambda \ln y) d \theta^{2}+d x_{3}^{2}, \\
F_{\theta} & =\lambda \sqrt{1+k},
\end{aligned}
$$

where for $k=0$ we recover the supersymmetric string soliton solution of [1] which is dual to the elementary string solution of Dabholkar et al [29]. The solution shown in (2.6) in fact represents a naked singularity, since the event horizon is pushed out to $r_{+}=\infty$, which agrees with the Horowitz-Strominger "no-4D-black-string" theorem [30].

Finally, we consider the two-parameter black membrane solution. In this case, we wrap the fivebrane around three of the compactified dimensions $\left(x_{7}, x_{8}, x_{9}\right)$. However, the four-dimensional action necessary to yield membrane solutions is not obtained by a simple dimensional reduction of the ten-dimensional action because of the non-vanishing of $F=H_{456}$. Instead, the effective action is obtained by treating $F^{2}$ as a cosmological constant and is given by

$$
S_{3}=\frac{1}{2 \kappa^{2}} \int d^{4} x \sqrt{-g} e^{-2 \Phi-3 \sigma_{3}}\left(R+4(\partial \Phi)^{2}+12 \partial \sigma_{3} \cdot \partial \Phi+6\left(\partial \sigma_{3}\right)^{2}-e^{6 \sigma_{3}} \frac{1}{2} F^{2}\right),
$$

In the case of the torus, $\sigma_{3}$ is the modulus field $g_{44}=g_{55}=g_{66}=e^{-2 \sigma_{3}}$. The twoparameter black membrane solution is then

$$
\begin{aligned}
e^{-2 \Phi} & =e^{2 \sigma_{3}}=\left(1-\frac{r}{r_{-}}\right) \\
d s^{2} & =-\left(1-\frac{r}{r_{+}}\right)\left(1-\frac{r}{r_{-}}\right)^{-1} d t^{2}+\left(1-\frac{r}{r_{+}}\right)^{-1}\left(1-\frac{r}{r_{-}}\right)^{-4} d r^{2}+d x_{2}^{2}+d x_{3}^{2}, \\
F & =-\left(r_{+} r_{-}\right)^{-1 / 2} .
\end{aligned}
$$

This solution represents a black membrane with event horizon at $r=r_{+}$and inner horizon at $r=r_{-}$. Changing coordinates via $y^{-1}=r^{-1}-r_{-}^{-1}$ and taking the extremal limit yields

$$
\begin{aligned}
e^{2 \Phi} & =e^{-2 \sigma_{3}}=\left(1+\frac{y}{r_{-}}\right) \\
d s^{2} & =-d t^{2}+d x_{2}^{2}+d x_{3}^{2}+e^{2 \Phi} d y^{2} \\
F & =-\frac{1}{r_{-}}
\end{aligned}
$$

which is just the supersymmetric domain wall solution [1]. 


\section{Consistency Check}

To verify that the above field configurations are indeed solutions, we recall the generic $D$-dimensional black $p$-branes of [2]. Consider an antisymmetric tensor potential of rank $d$, $A_{\mu_{1}, \ldots, \mu_{d}}$, in $D$ spacetime dimensions $(\mu=0,1, \ldots,(D-1))$ interacting with the canonical Einstein metric $g_{\mu \nu}(\mathrm{can})$ and a scalar field $\phi$ via the action

$$
I_{D}(d)=\frac{1}{2 \kappa^{2}} \int d^{D} x \sqrt{-g}\left(R-\frac{1}{2}(\partial \phi)^{2}-\frac{1}{2(d+1) !} e^{-\alpha \phi} F_{\mu_{1} \mu_{2} \ldots \mu_{d+1}} F^{\mu_{1} \mu_{2} \ldots \mu_{d+1}}\right)
$$

where

$$
\alpha^{2}(d)=4-\frac{2 d \tilde{d}}{d+\tilde{d}}
$$

and where we have introduced the dual worldvolume dimension $\tilde{d}$ via

$$
\tilde{d}=D-d-2
$$

To understand this choice of $\alpha$, we recall the action for an elementary $d$-dimensional extended object (a "( $d-1)$-brane") with worldvolume coordinates $\xi^{i}(i=1,2, \ldots, d-1)$, metric $\gamma_{i j}(\xi)$ and tension $T_{d}$, coupled to the $D$-dimensional background fields $g_{\mu \nu}(\operatorname{can})$, $A_{\mu_{1} \cdots \mu_{d}}$ and $\phi:$

$$
\begin{aligned}
S_{d}=T_{d} \int d^{d} \xi & \left(-\frac{1}{2} \sqrt{-\gamma} \gamma^{i j} \partial_{i} \mathrm{X}^{\mu} \partial_{j} \mathrm{X}^{\nu} g_{\mu \nu} e^{\alpha \phi / d}+\frac{(d-2)}{2} \sqrt{-\gamma}\right. \\
& \left.-\frac{1}{d !} \epsilon^{i_{1} i_{2} \cdots i_{d}} \partial_{i_{1}} \mathrm{X}^{\mu_{1}} \partial_{i_{2}} \mathrm{X}^{\mu_{2}} \cdots \partial_{i_{d}} \mathrm{X}^{\mu_{d}} A_{\mu_{1} \cdots \mu_{d}}\right)
\end{aligned}
$$

The above action is the bosonic sector of a spacetime supersymmetric, $\kappa$-symmetric, GreenSchwarz action for the super $p$-brane. In particular, the crucial relative coefficient of the Wess-Zumino and kinetic terms (the "charge to mass ratio") is fixed by $\kappa$-symmetry. The physical significance of the choice of $\alpha$ given in (3.2) is that it is the one singled out by

the requirement that the combined system $I_{D}(d)+S_{d}$ admits elementary $(d-1)$-brane solutions [2] with mass per unit $p$-volume, $\mathcal{M}_{d}$, and Noether "electric" charge, $e_{d}$, given by $\sqrt{2} \kappa \mathcal{M}_{d}=e_{d}$. These extreme solutions preserve half the spacetime supersymmetries since this charge-to-mass ratio is the one singled out by $\kappa$-symmetry.

Having fixed the choice of $\alpha$, we now look for black $(\tilde{d}-1)$-brane solutions of $I_{D}(d)$ alone, carrying a topological "magnetic" charge $g_{\tilde{d}}$ obeying the Dirac quantization rule

$$
e_{d} g_{\tilde{d}}=2 \pi n, \quad n=\text { integer }
$$


The solutions are [2] (with $i=1, \ldots, \tilde{d}-1)$ :

$$
\begin{aligned}
e^{-2 \phi} & =\Delta_{-}^{\alpha}, \\
d s^{2} & =-\Delta_{+} \Delta_{-}^{\frac{-\tilde{d}}{(d+\tilde{d})}} d t^{2}+\Delta_{+}^{-1} \Delta_{-}^{\frac{\alpha^{2}}{2 d}-1} d r^{2}+r^{2} \Delta_{-}^{\frac{\alpha^{2}}{2 d}} d \Omega_{d+1}^{2}+\Delta_{-}^{\frac{d}{(d+\tilde{d})}} d x^{i} d x_{i}, \\
F_{d+1} & =d\left(r_{+} r_{-}\right)^{d / 2} \epsilon_{d+1} .
\end{aligned}
$$

where $\Delta_{ \pm}=\left[1-\left(\frac{r_{ \pm}}{r}\right)^{d}\right]$ and $\epsilon_{n}$ the volume form on $S^{n}$. The magnetic charge $g_{\tilde{d}}$ and the mass per unit volume $\mathcal{M}_{\tilde{d}}$ are related to $r_{ \pm}$by

$$
\begin{aligned}
g_{\tilde{d}} & =\frac{\Omega_{d+1}}{\sqrt{2} \kappa} d\left(r_{-} r_{+}\right)^{\frac{d}{2}}, \\
\mathcal{M}_{\tilde{d}} & =\frac{\Omega_{d+1}}{2 \kappa^{2}}\left((d+1) r_{+}^{d}-r_{-}^{d}\right)
\end{aligned}
$$

where $\Omega^{n}$ is the volume of $S^{n}$. In the extremal limit, $r_{-}=r_{+}$, the solutions saturate the Bogomol'nyi bound $\sqrt{2} \kappa \mathcal{M}_{\tilde{d}}=g_{\tilde{d}}$, and also preserve half the spacetime supersymmetry. In this limit, the line element reduces to (with $\beta=0, \ldots, \tilde{d}-1$ )

$$
d s^{2}=\Delta_{-}^{\frac{d}{(d+\tilde{d})}} d x^{\beta} d x_{\beta}+\Delta_{-}^{\frac{\alpha^{2}}{2 d}}\left(\Delta_{-}^{-2} d r^{2}+r^{2} d \Omega_{d+1}^{2}\right)
$$

and, for $\alpha \neq 0$, exhibits a curvature singularity at $r=r_{-}$. However, as discussed in [2,3], when expressed in terms of the dual $(d-1)$-brane $\sigma$-model metric $g_{\mu \nu}(\sigma-$ model $)=$ $e^{\alpha \phi / d} g_{\mu \nu}(\operatorname{can})$, we find

$$
d s^{2}=\Delta_{-}^{\frac{(d-2)}{d}} d x^{\beta} d x_{\beta}+\left(\Delta_{-}^{-2} d r^{2}+r^{2} d \Omega_{d+1}^{2}\right)
$$

which is regular at $r=r_{-}$. So these extremal black $p$-branes are non-singular when viewed as solutions of the dual theory. They are also non-singular in the sense that the proper time taken for a test $p$-brane to fall radially into a dual source $\tilde{p}$-brane is infinite, and vice versa [3] (assuming $p, \tilde{p} \geq 0)$.

The $\sigma$-model metric is, by definition, the one for which $S_{d}$ is independent of $\phi$. Regarding $S_{d}$ as a "matter Lagrangian" therefore defines a Brans-Dicke type theory with parameter [2]

$$
\omega=-\frac{(D-1)(d-2)-d^{2}}{(D-2)(d-2)-d^{2}}
$$

We now wish to demonstrate that the black hole, string and domain wall solutions of the heterotic string discussed in section 1 are nothing but the $(\tilde{d}=1, d=1, \alpha=\sqrt{3}, \omega=$ 
$-4 / 3),(\tilde{d}=2, d=0, \alpha=2, \omega=-3 / 2)$ and $(\tilde{d}=3, d=-1, \alpha=\sqrt{7}, \omega=-10 / 7)$ special cases of the above solutions ${ }^{\dagger}$. To confirm this, it is sufficient to show that the three actions $S_{1}, S_{2}$ and $S_{3}$ may be cast into the form (3.1). This is achieved by transforming the metric $g_{\mu \nu}$ and scalars $\Phi$ and $\sigma_{i},(i=1,2,3)$, to the canonical metric $g_{\mu \nu}($ can $)$ and scalars $\phi$ and $\lambda$ via the following field redefinitions:

monopole

$$
\begin{aligned}
g_{\mu \nu} & =e^{\frac{1}{\sqrt{3}}(\sqrt{2} \lambda-\phi)} g_{\mu \nu}(\mathrm{can}), \\
\Phi & =\frac{1}{2 \sqrt{3}}\left(\frac{\lambda}{\sqrt{2}}-2 \phi\right), \\
\sigma_{1} & =\frac{1}{\sqrt{3}}\left(\frac{\lambda}{\sqrt{2}}+\phi\right)
\end{aligned}
$$

string

$$
\begin{aligned}
g_{\mu \nu} & =e^{\lambda} g_{\mu \nu}(\mathrm{can}), \\
\Phi & =\frac{1}{2}(\lambda-\phi), \\
\sigma_{2} & =\frac{1}{2} \phi
\end{aligned}
$$

membrane

$$
\begin{aligned}
g_{\mu \nu} & =e^{\frac{1}{\sqrt{7}}(\sqrt{6} \lambda+\phi)} g_{\mu \nu}(\text { can }), \\
\Phi & =\frac{1}{2 \sqrt{7}}\left(\frac{3 \sqrt{3}}{\sqrt{2}} \lambda-2 \phi\right), \\
\sigma_{3} & =\frac{1}{\sqrt{7}}\left(-\frac{\lambda}{\sqrt{6}}+\phi\right)
\end{aligned}
$$

Having done this, we can then set $\lambda=0$ to obtain the desired result. Note that by analytically continuing the solution (3.6) to the cases $d=0$ and $d=-1$, we are extrapolating the meaning of the ADM mass and topological charge to non-asymptotically flat spacetimes.

\section{4. $\alpha=\sqrt{3}$ in the Type II String: Electric/Magnetic Duality in $D=4$ from Particle/Sixbrane Duality in $D=10$}

We note that the black hole solution corresponds to a Maxwell-scalar coupling $e^{-\alpha \phi} F_{\mu \nu} F^{\mu \nu}$ with $\alpha=\sqrt{3}$. This is to be contrasted with the pure dilaton black hole solutions of the heterotic string that have attracted much attention recently [1 13 and

$\dagger$ This means, in particular, that the extreme magnetic monopole will take an infinite proper time to fall radially into the extreme electric monopole, and vice versa. 
have $\alpha=1^{\dagger}$. The case $\alpha=\sqrt{3}$ also occurs when the Maxwell field and the scalar field $\phi$ arise from a Kaluza-Klein reduction of pure gravity from $D=5$ to $D=4$ :

$$
\hat{g}_{M N}=e^{\frac{\phi}{\sqrt{3}}}\left(\begin{array}{cc}
g_{\mu \nu}+e^{-\sqrt{3} \phi} A_{\mu} A_{\nu} & e^{-\sqrt{3} \phi} A_{\mu} \\
e^{-\sqrt{3} \phi} A_{\nu} & e^{-\sqrt{3} \phi}
\end{array}\right)
$$

where $\hat{g}_{M N}(M, N=0,1,2,3,4)$ and $g_{\mu \nu}(\mu, \nu=0,1,2,3)$ are the canonical metrics in 5 and 4 dimensions respectively. The resulting action is given by

$$
S=\frac{1}{2 \kappa^{2}} \int d^{4} x \sqrt{-g}\left[R-\frac{1}{2}(\partial \phi)^{2}-\frac{1}{4} e^{-\sqrt{3} \phi} F_{\mu \nu} F^{\mu \nu}\right]
$$

and it admits as an "elementary" solution the $\alpha=\sqrt{3}$ black hole metric (2.2), but with the scalar field

$$
e^{-2 \phi}=\Delta_{-}^{\sqrt{3}}
$$

and the electric field

$$
\frac{1}{\sqrt{2} \kappa} e^{-\sqrt{3} \phi *} F_{\theta \varphi}=\frac{e}{4 \pi} \sin \theta
$$

corresponding to an electric monopole with Noether charge $e$. This system also admits the topological magnetic solution with

$$
e^{-2 \phi}=\Delta_{-}^{-\sqrt{3}}
$$

and the magnetic field

$$
\frac{1}{\sqrt{2} \kappa} F_{\theta \varphi}=\frac{g}{4 \pi} \sin \theta
$$

corresponding to a magnetic monopole with topological magnetic charge $g$ obeying the Dirac quantization rule

$$
e g=2 \pi n, n=\text { integer }
$$

In effect, it was for this reason that the $\alpha=\sqrt{3}$ black hole was identified as a solution of the Type II string in [2], the fields $A_{\mu}$ and $\phi$ being just the abelian gauge field and the dilaton of $(N=2, D=10)$ supergravity which arises from Kaluza-Klein compactification of $(N=1, D=11)$ supergravity.

$\dagger$ Contrary to some claims in the literature, the pure Reissner-Nordström black hole with $\alpha=0$ is also a solution of the low energy heterotic string equations. This may be seen by noting that it provides a solution to $(N=2, D=4)$ supergravity which is a consistent truncation of toroidally compactified $N=1, D=10$ supergravity [31]. 
Some time ago, Gibbons and Perry [19] pointed out that $N=8$ supergravity, compactified from $D=5$ to $D=4$, admits an infinite tower of elementary states with mass $m_{n}$ and electric charge $e_{n}$ given by $\sqrt{2} \kappa m_{n}=e_{n}$, where $e_{n}$ are quantized in terms of a fundamental charge $e, e_{n}=n e$, and that these elementary states fall into $N=8$ supermultiplets. They also pointed out that this theory admits an infinite tower of solitonic states with the masses $\tilde{m}_{n}$ and magnetic charge $g_{n}$ given by $\sqrt{2} \kappa \tilde{m}_{n}=g_{n}=n g$, where $e$ and $g$ obey eg $=2 \pi$, which also fall into the same $N=8$ supermultiplets. They conjectured, á la Olive-Montonen [32], that there should exist a dual formulation of the theory for which the roles of electric elementary states and magnetic solitonic states are interchanged. It was argued in [2] that this electric/magnetic duality conjecture in $D=4$ could be reinterpreted as a particle/sixbrane duality conjecture in $D=10$.

To see this, consider the action dual to $S$, with $\alpha=-\sqrt{3}$, for which the roles of Maxwell field equations and Bianchi identities are interchanged:

$$
\tilde{S}=\frac{1}{2 \kappa^{2}} \int d^{4} x \sqrt{-g}\left[R-\frac{1}{2}(\partial \phi)^{2}-\frac{1}{4} e^{\sqrt{3} \phi} \tilde{F}_{\mu \nu} \tilde{F}^{\mu \nu}\right],
$$

where

$$
\tilde{F}_{\mu \nu}=e^{-\sqrt{3} \phi *} F_{\mu \nu}
$$

This is precisely the action obtained by double dimensional reduction of a dual formulation of ( $D=10, N=2)$ supergravity in which the two-form $F_{M N}(M, N=0, \ldots, 9)$ is swapped for an 8-form $\tilde{F}_{M_{1} . . M_{8}}$, where $\tilde{F}_{\mu \nu}=\tilde{F}_{\mu \nu 456789}$. This dual action also admits both electric and magnetic monopole solutions but because the roles of field equations and Bianchi identities are interchanged, so are the roles of electric and magnetic. Since the 1-form and 7 -form potentials, which give rise to these 2 -form and 8 -form field strengths, are those that couple naturally to the worldline of a point particle or the worldvolume of a 6 -brane, we see that the Gibbons-Perry $(N=8, D=4)$ electric/magnetic duality conjecture may be re-expressed as an (Type II, $D=10$ ) particle/sixbrane duality conjecture. Indeed, the Horowitz-Strominger $D=10$ black sixbrane [30] is simply obtained by adding 6 flat dimensions to the $D=4, \alpha=\sqrt{3}$ magnetic black hole.

In general, the $N=8$ theory will admit black holes with $\alpha=0,1$ and $\sqrt{3}$ whose extreme limits preserve 1,2 or 4 spacetime supersymmetries, respectively. Defining $\mathcal{M}_{1}=$ $M, g_{1}^{2}=4 \pi Q^{2}$ and $\kappa^{2}=8 \pi G$, these extreme black holes satisfy the "no-force" condition, i.e. they saturate the Bogomol'nyi bounds

$$
G\left(M^{2}+\Sigma^{2}\right)=\left(1+\alpha^{2}\right) G M^{2}=N^{\prime} G M^{2}=Q^{2}
$$

where $\Sigma=\alpha M$ is the scalar charge and $N^{\prime}$ is the number of unbroken supersymmetries. 


\section{5. $\alpha=\sqrt{3}$ in the Heterotic String: Electric/Magnetic Duality in $D=4$ from String/Fivebrane Duality in $D=10$}

The results of the present paper now allow us to discuss the $\alpha=\sqrt{3}$ electric/magnetic duality from a totally different perspective from that in [2] and section 4 . For concreteness, let us focus on generic toroidal compactification of the heterotic string. Instead of the $N=8$ supergravity of section 4 , the four-dimensional theory is now $N=4$ supergravity coupled to $22 N=4$ vector multiplets ${ }^{\dagger}$. The same dual Lagrangians (4.2) and (4.8) still emerge but with completely different origins. The Maxwell field $F_{\mu \nu}$ (or $\tilde{F}_{\mu \nu}$ ) and the scalar field $\phi$ do not come from the $D=10$ 2-form (or 8-form) and dilaton of the Type II particle (or sixbrane), but rather from the $D=103$-form (or 7-form) and dilaton plus modulus field of the heterotic string (or heterotic fivebrane). Thus, the $D=4$ electric/magnetic duality can now be re-interpreted as a $D=10$ string/fivebrane duality!

Because of the non-vanishing modulus field $g_{44}=e^{-2 \sigma}$ however, the $D=10$ black fivebrane solution is not obtained by adding 6 flat dimensions to the $D=4$ black hole. Rather the two are connected by wrapping the fivebrane around 5 of the 6 extra dimensions [1].

The possibility of a heterotic stringy explanation of four-dimensional electric/magnetic duality has also been considered by Sen [33 35]. But his notion of duality is different from the one considered here. The compactified heterotic string displays a target space duality $O(6,22, Z)$. It is also conjectured to display the strong/weak coupling $S L(2, Z)$ $S$-duality relating the dilaton and the axion, which is certainly there in the field theory limit. See [36] for a recent review. The "duality of dualities" suggestion [37 39, [1] is that, under string/fivebrane duality, the roles of $S$ and $T$ dualities are interchanged. Thus Sen's duality refers to $S L(2, Z) S$ duality, whereas ours refers to $S \leftrightarrow T$. A similar distinction is to be drawn between the two uses of the words "dual string" [34, 1]. In any event, the picture that emerges is one in which the massive states of the string correspond to extreme black holes.

$\dagger$ Gibbons discusses both the $\alpha=1$ black hole of pure $N=4$ supergravity and the $\alpha=\sqrt{3}$ Kaluza-Klein black hole in the same paper [4], as does Horowitz [1]. Moreover, black holes in pure $N=4$ supergravity are treated by Kallosh et al. [8 10]. The reader may therefore wonder why the $\alpha=\sqrt{3} N=4$ black hole discussed in the present paper was overlooked. The reason is that pure $N=4$ supergravity does not admit the $\alpha=\sqrt{3}$ solution; it is crucial that we include the $N=4$ vector multiplets in order to introduce the modulus fields. 


\section{Conclusion}

Of course, the present paper has established only that these two-parameter configurations are solutions of the field theory limit of the heterotic string. Although the extreme one-parameter solutions are expected to be exact to all orders in $\alpha^{\prime}$, the same reasoning does not carry over to the new two-parameter solutions. It would be interesting to pursue conformal field theory arguments, perhaps along the lines recently suggested by Giddings, Polchinski and Strominger [13].

It would be also interesting to see whether the generalization of the one-parameter solutions of [1] to the two-parameter solutions of the present paper can be carried out when we include the Yang-Mills coupling as in [1]. This would necessarily involve giving up the self-duality condition on the Yang-Mills field strength, however, since the self-duality condition is tied to the extreme, $\sqrt{2} \kappa \mathcal{M}_{p+1}=g_{p+1}$, supersymmetric solutions.

Finally, there is the question of whether these solutions are peculiar to the toroidal compactification or whether they survive in more realistic orbifold or Calabi-Yau models [40]. Although the actions $S_{1}, S_{2}$ and $S_{3}$ were originally derived in the context of the torus [1], they also appear in a large class of $N=1$ supergravity theories.

\section{Acknowledgments}

We have enjoyed useful conversations with Ergin Sezgin. 


\section{References}

[1] M. J. Duff and R. R. Khuri, CTP/TAMU-17/93 (hep-th/9305142) (to appear in Nucl. Phys. B).

[2] M. J. Duff and J. X. Lu, CTP/TAMU-54/92 (hep-th@9306052).

[3] M. J. Duff, R. R. Khuri and J. X. Lu, Nucl. Phys. B377 (1992) 281.

[4] G. W. Gibbons, Nucl. Phys. B207 (1982) 337.

[5] G. W. Gibbons and K. Maeda, Nucl. Phys. B298 (1988) 741.

[6] D. Garfinkle, G. T. Horowitz and A. Strominger, Phys. Rev. D43 (1991) 3140.

[7] A. Shapere, S. Trivedi and F. Wilczek, Mod. Phys. Lett. A6 (1991) 2677.

[8] R. Kallosh, A. Linde, T. Ortin, A. Peet and A. Van Proeyen, Phys. Rev. D46 (1992) 5278.

[9] R. Kallosh, SU-ITP-92-1 (hep-th@9201029).

[10] R. Kallosh and T. Ortin, SU-ITP-93-3 (hep-th@9302109).

[11] G. T. Horowitz, UCBTH-92-32 (hep-th@9210119).

[12] A. Sen, TIFR-TH-92-57 (hep-th@9210050).

[13] S. B. Giddings, J. Polchinski and A. Strominger, NSF-ITP-93-62 (hep-th@9305083).

[14] P. Dobiasch and D. Maison, Gen. Rel. Grav. 14 (1982) 231.

[15] A. Chodos and S. Detweiler, Gen. Rel. Grav. 14 (1982) 879.

[16] D. Pollard, J. Phys. A16 (1983) 565.

[17] D. J. Gross and M. J. Perry, Nucl. Phys. B226 (1983) 29.

[18] R. D. Sorkin, Phys. Rev. Lett. 51 (1983) 87.

[19] G. W. Gibbons and M. J. Perry, Nucl. Phys. B248 (1984) 629.

[20] C. F. E. Holzhey and F. Wilczek, Nucl. Phys. B360 (1992) 447.

[21] M. J. Duff, Class. Quantum Grav. 5 (1988) 189.

[22] A. Strominger, Nucl. Phys. B343 (1990) 167.

[23] M. J. Duff and J. X. Lu, Nucl. Phys. B354 (1991) 129.

[24] E. Cremmer, S. Ferrara, L. Girardello, A. Van Proeyen, Nucl. Phys. B212 (1983) 413.

[25] R. R. Khuri, Phys. Lett. B259 (1991) 261.

[26] R. R. Khuri, Phys. Lett. B294 (1992) 325.

[27] R. R. Khuri, Nucl. Phys. B387 (1992) 315.

[28] J. P. Gauntlett, J. A. Harvey and J. T. Liu, EFI-92-67, IFP-434-UNC (hepth@9211056).

[29] A. Dabholkar, G. Gibbons, J. A. Harvey, F. R. Ruiz, Nucl. Phys. B340 (1990) 33

[30] G. T. Horowitz, A. Strominger, Nucl. Phys. B360 (1991) 2930.

[31] M. J. Duff and C. N. Pope, Nucl. Phys B255 (1985) 355.

[32] C. Montonen and D. Olive, Phys. Lett. B72 (1977) 117.

[33] A. Sen, Nucl. Phys. B404 (1993) 109.

[34] A. Sen, TIFR-TH-93-03 (hep-th@9302038). 
[35] A. Sen, Phys. Lett. B303 (1993) 22.

[36] J. H. Schwarz, CALT-68-1879 (hep-th@9307121).

[37] J. H. Schwarz and A. Sen, NSF-ITP-93-46, CALT-68-1863, TIFR-TH-93-19 (hepth@9304154).

[38] P. Binetruy, Phys. Lett 315 (1993) 80.

[39] J. H. Schwarz and A. Sen, Phys. Lett. B312 (1993) 105

[40] R. D'Auria, S. Ferrara and M. Villasante, CERN-TH 6914/93, POLFIS-TH 04/93, UCLA/93/TEP/18 (hep-th@9306125). 